
\title{
$\begin{array}{ll}\text { Research Square } & \begin{array}{l}\text { Preprints are preliminary reports that have not undergone peer review. } \\ \text { They should not be considered conclusive, used to inform clinical practice, } \\ \text { or referenced by the media as validated information. }\end{array}\end{array}$
}

\section{Aspirin Regulates Gemcitabine Resistance in Pancreatic Cancer via Inhibiting the PI3K/Akt/mTOR Signaling Pathway and Reversing Epithelial- mesenchymal Transition}

\section{hanyu zhou}

Department of Oncology, The Second People's Hospital of Changzhou, Nanjing Medical University, Changzhou, Jiangsu 213003, P.R. China

\section{ZhiYing Yan}

Department of Oncology, The Affiliated Changzhou No.2 People's Hospital of Nanjing Medical University Feifei Wei

Department of Oncology, The Affiliated Changzhou No.2 People's Hospital of Nanjing Medical University Muhan Liu the afiliated changzhou no.2 people's hospital of nanjing medical university

yi liu

Department of Oncology, The Second People's Hospital of Changzhou, Nanjing Medical University, Changzhou, Jiangsu 213003, P.R. China

\section{Xianming Zhang}

Department of Oncology, The Affiliated Changzhou No.2 People's Hospital of Nanjing Medical University Kequn xu ( $\sim 13775001122 @ 139 . c o m)$

The Affiliated Changzhou No 2 People's Hospital of Nanjing Medical University https://orcid.org/00000003-3782-0152

\section{Yingwei Zhu}

Department of Oncology, The Affiliated Changzhou No.2 People's Hospital of Nanjing Medical University xiao yun

The Affiliated Changzhou No 2 People's Hospital of Nanjing Medical University

\section{Research}

Keywords: aspirin, PI3K/Akt/mTOR signaling pathway, EMT, chemoresistance, pancreatic cancer

Posted Date: August 23rd, 2021

DOl: https://doi.org/10.21203/rs.3.rs-818856/v1 
License: (c) (i) This work is licensed under a Creative Commons Attribution 4.0 International License. Read Full License 


\section{Abstract}

Background: Gemcitabine is considered a classical agent for the treatment of patients with pancreatic cancer. However, gemcitabine resistance is a common cause of treatment failure, leading to poor survival. Therapy to overcome gemcitabine resistance would benefit patients with pancreatic cancer. This study investigated the impact of aspirin (ASA) to gemcitabine resistance in the biological function of pancreatic cancer cells and the potential mechanism.

Methods: The MTT assay, wound healing assay and annexin V-FITC/PI test was used to determine whether ASA could inhibit gemcitabine resistance in pancreatic cancer cells. Besides, the expression of Bcl-2, Bax, E-cadherin, Vimentin, p-PI3K, p-AKT and p-mTOR was detected by the Western blot. Statistically significant differences between groups were determined with the Student's t-test.

Results: The proliferation and migration of SW1990 and BxPC3 cells were significantly decreased while the apoptosis rate was increased in the combination of ASA and gemcitabine group $(p<0.05)$. The level of $\mathrm{Bcl}-2$ was decreased and the level of Bax was increased significantly in the combination of ASA and gemcitabine group $(p<0.05)$ while the level of $\mathrm{p}-\mathrm{PI} 3 \mathrm{~K}, \mathrm{p}$-AKT and $\mathrm{p}-\mathrm{mTOR}$ was decreased $(p<0.05)$. What's more, this group significantly reversed EMT in SW1990 and BXPC3 cells with an increase in the expression of E-cadherin and a decrease in Vimentin.

Conclusion: Our research provided evidence ASA could help to inhibit gemcitabine resistance of pancreatic cancer cells, probably via inhibiting the PI3K/AKT/mTOR pathway and reversing EMT. Thus, combined use of ASA and gemcitabine is expected to be a potential therapeutic strategy for pancreatic cancer patients.

\section{Introduction}

Pancreatic cancer is the eighth leading cause of cancer deaths worldwide with early metastasis and a very poor prognosis. The overall 5-year survival rate is less than 9\% [1]. At diagnosis, most patients present with local regional spread and/or distant metastasi [2]. At present, gemcitabine is recognized by many oncologists as the first-line agent for the treatment of pancreatic cancer. However, the monotherapy with gemcitabine tends to develop acquired resistance in pancreatic cancer, which becomes a major concern in clinical practice [3-5] , and the underlying mechanism of chemo-resistance is poorly understood.

The PI3K/AKT/mTOR signaling pathway has been proved important in tumor development and is often activated in pancreatic cancer (PC) [6]. AKT has been proved to contribution to cancer development, which is activated by PI3K, growth factors and so on. Downstream effectors like mTOR lead to signal transduction [7]. PI3K is a downstream effector of oncogenic KRAS, which is nearly ubiquitous in pancreatic cancer [8]. A recent study which analyzed 32 cancer types in The Cancer Genome Atlas (TCGA) has identified KRAS exert a strong pro-tumorigenic effect through PI3K/AKT/mTOR pathway 
activation in pancreatic cancer [9]. A recent study shows mTOR activation is connected with gemcitabine resistance in pancreatic cancer [10].

The highly aggressive feature of pancreatic cancer may be partly attributed to the chemotherapy-resistant characteristics of pancreatic cancer cells which is related to the epithelial-mesenchymal transition (EMT) phenotype and cancer stem cells [11]. The EMT is an orchestrated series of events leading to altered cellcell and cell-extra cellular matrix interactions that transform the cancer tissue with a fibroblast-like morphology, and confer migratory and invasive properties on neoplastic cells $[12,13]$. In essence, EMT represents the transition between two fully differentiated and mature cells [14]. In tumor cells, the expression of phenotype marker proteins of epithelial cells (e.g. E-cadherin) is decreased, and the expression of phenotype marker proteins of mesenchymal cells (e.g. Vimentin and N-cadherin) is increased. Homocellular tight junction and cell polarity are also deficient $[15,16]$. Importantly, some research indicated the EMT may alter the sensitivity of neoplastic cells to chemotherapy agents $[17,18]$. Therefore, target therapy against or minimizing EMT may increase the efficacy of chemotherapy.

As we all know, several kinds of chemotherapeutic agents are now widely used in the first line including $5 F U$, irinotecan, oxaliplatin and taxanes. When it comes to the reason why we specifically choose gemcitabine for our study, here are our answer. According to National Comprehensive Cancer Network (NCCN) Guidelines Version 1.2019 Pancreatic Adenocarcinoma, gemcitabine, is contained in first-line therapy or preferred regimens of neoadjuvant therapy (resectable/borderline resectable disease), adjuvant therapy, locally advanced disease and metastatic disease. In a word, gemcitabine is the standard first-line chemotherapeutic drug of pancreatic cancer. In the first line, FOLFIRINOX which including 5FU, irinotecan, oxaliplatin and taxanes is also significant. However, FOLFIRINOX is not recommended in poor performance status. Moreover, almost all the patients came across several side effects when treated with FOLFIRINOX, which placed restrictions on adding aspirin. As we know, adding aspirin in a therapy may increase risk of bleeding which is rather serious and could lead to death in cancer patients while anemia is common. That's part of the reasons why we didn't combine aspirin with FOLFIRINOX which including 5FU, irinotecan, oxaliplatin and taxanes. Additionally, it was reported aspirin inhibits proliferation of pancreatic cancer cells via inhibiting PI3K/Akt/mTOR signaling pathway. Moreover, aspirin also shows a therapeutic potential through targeted inhibition on tumor growth and angiogenesis $[19,20]$. Many studies pointed out gemcitabine activation of Akt/mTOR signaling pathway in resistant cells is a key signaling event for gemcitabine resistance, and we suppose aspirin may resensitize resistant cells to gemcitabine by inhibiting this activated pathway [21-23]. Considering the above two points, when it comes to combining with aspirin, gemcitabine may be more suitable for research than $5 F U$, irinotecan, oxaliplatin, taxanes or other chemotherapeutic agents.

ASA, a weak organic acid, show promise as cancer chemoprevention agents due to their antiinflammatory properties [24]. A pooled analysis of 25,570 patients in eight trials recently reported that daily aspirin use reduced deaths of several common cancers, including pancreatic cancer deaths, with most benefit seen after 5 years of the scheduled treatment [25], while a clinic-based case-control study showed that aspirin use is associated with lowered risk of developing pancreatic cancer [26]. Several 
years ago, Streicher SA, et al. conducted a study in Connecticut and found approximately $50 \%$ reduced risk of pancreatic cancer with regular use of either low-dose or regular-dose aspirin. In China, a population-based study of 761 case and 794 control subjects was performed to show ever-regular use of aspirin was associated with lowered risk of pancreatic cancer: odds ratio [OR] $=0.54 ; 95 \% \mathrm{Cl}$ : 0.40-0.73. However, the evidence that aspirin use may lower risk of pancreatic cancer is still full of conflicts around the world and the role of ASA in the gemcitabine resistance of pancreatic cancer remain ambiguous, as well as the molecular mechanism, if ASA really benefits.

\section{Materials And Methods \\ Cell lines and cell culture}

Human pancreatic cancer cell lines SW1990 and BxPC3 were obtained from the Shanghai Cell Bank of the Chinese Academy of Sciences and the Changhai hospital affiliated to the Second Military Medical University. Mycoplasma testing has been done for the cell lines used. Cells were cultured in 1640 medium (Gibco, Gaithersburg, MD, USA) containing 10\% fetal bovine serum (FBS), and maintained in a humid atmosphere at $37^{\circ} \mathrm{C}$ with $5 \% \mathrm{CO}^{2}$. The cell medium was changed every 2 to 3 days. Cell passage was performed with trypsin when the confluence of monolayer cells reached $70 \%$ to $80 \%$.

\section{Main reagents}

ASA was purchased from Sigma (St. Louis, MO, USA) and was dissolved in DMSO (Dimethyl sulfoxide), which was obtained from $S R L$, India, to make a $5 \mathrm{M}$ stock solution. This solution was stored at $-20^{\circ} \mathrm{C}$. Gemcitabine was obtained from the Lilly Pharmaceuticals (Indianapolis, IN, USA) and dissolved in a sterile saline solution to create a $5 \mathrm{~g} / \mathrm{L}$ stock solution. 3-(4,5-dimethylthiazol-2-yl)-2,5-diphenyltetra-zolium bromide (MTT) (St. Louis, MO, USA) was set to $5 \mathrm{mg} / \mathrm{ml}$. The Annexin V-FITC apoptosis detection kit was obtained from R\&D (Minneapolis, MN, USA). The rabbit monoclonal antibody against human E-cadherin, Vimentin, Bcl-2, Bax, PI3K, p-PI3K, Akt, p-Akt, mTOR, p-mTOR and $\beta$-actin was obtained from the Santa Cruz Biotechnology (Santz Cruz, CA, USA). The rabbit monoclonal antibody against human Vimentin was obtained from Cell Signaling Technology (Boston, MA, USA). Horseradish peroxidase-labeled (HRP) goatanti-rabbit IgG (secondary antibody) antibodies were obtained from CWBIO (Beijing ComWin Biotech, China).

\section{Cell proliferation assay}

Cells were cultured in 1640 medium containing $10 \% \mathrm{FBS}$, and maintained in a humid atmosphere at $37^{\circ} \mathrm{C}$ with $5 \% \mathrm{CO}^{2}$ for $72 \mathrm{~h}$. Then cells were harvested and inoculated into 96 -well plates with $3 \times 10^{3}$ cells in each well $24 \mathrm{~h}$ before treatment. After that, samples were divided into 4 groups. Cells were cultured in: 1)1640 medium containing 10\% FBS and 2 mmol/L ASA, 2)1640 medium containing 10\% FBS and 0.05 
$\mathrm{mg} / \mathrm{L}$ gemcitabine, 3) 1640 medium containing $10 \% \mathrm{FBS}, 2 \mathrm{mmol} / \mathrm{L}$ ASA and $0.05 \mathrm{mg} / \mathrm{L}$ gemcitabine, 4) negative control (1640 medium containing 10\% FBS). 24 hours later, we incubated cells at $37^{\circ} \mathrm{C}$ with $5 \%$ $\mathrm{CO}^{2}$ for next 0 hours, 24 hours, 48 hours and 72 hours. Then, $10 \% \mathrm{MTT}(5 \mathrm{mg} / \mathrm{mL})$ was added into each well. After adding MTT for 4 hours, total of $150 \mu$ DMSO was then added into each well, and the samples were vibrated for 10 minutes. The absorbance (OD) was then measured with a microplate reader set at $570 \mathrm{~nm}$. Each cell proliferation experiment was repeated 3 times and the readings were averaged for the statistical analysis.

\section{Wound Healing Assay}

BxPC3 and SW1990 cells were seeded in 6-well plates. When the cell confluence was about $100 \%$, scratches were made on the monolayer cell surface with 200- $\mu$ l pipette tips. After the cells were washed twice with PBS, the cells were divided into 4 groups, which was mentioned in the cell proliferation assay. Images of cells on both sides of the scratches were captured with an inverted microscope at 0 and 2 hours. Finally, images have analyzed the migration in three fields of view that were randomly selected and analyzed in an independent trial with 400X magnification under an inverted microscope (Olympus Corp). The width (W) of the scratch measured; the percentage of wound area remaining was calculated as $\mathrm{W}_{24 \mathrm{~h}} / \mathrm{W}_{0 \mathrm{~h}} \times 100 \%$. All experiments were performed in triplicate

\section{Cell apoptosis}

BxPC3 and SW1990 cells were seeded in 6-well plates and cultured with 1640 medium containing 10\% FBS. After the cells were attached to the plate for $24 \mathrm{~h}$, they were divided into 4 groups, which was mentioned in the cell proliferation assay, and cultured for $24 \mathrm{~h}$. The cells were then collected, washed with phosphate buffer saline (PBS) solution 2 to 3 times. To count the number of apoptotic cells, they were stained with annexin V-fluorescein isothiocyanate (FITC)/propidium iodide (PI). These cells were analyzed by using the BD FACSCalibur in each experiment. All experiments were carried out in triplicate.

\section{Western blot Analysis}

The 4 groups cells, which was mentioned in the cell proliferation assay, were harvested after cultured for $24 \mathrm{~h}$ and lysed with RIPA lysis buffer. Protein molecules released from the cells were separated and extracted by the SDS-PAGE (Sodium dodecyl sulfate-Polyacrylamide gel electrophoresis), and then transferred to polyvinylidene fluoride (PVDF) membrane for western blotting. Membranes were blocked with $5 \%$ non-fat milk and then incubated with primary antibodies against $\beta$-actin, Bax, Bcl-2, E-cadherin, Vimentin, PI3K, phospho-PI3K, Akt, phospho-Akt, mTOR and phospho-mTOR at the dilution of 1:1000 at $4^{\circ} \mathrm{C}$ overnight. The membranes were then rewarmed for 30 minutes, washed with TBST (Tris Buffered saline Tween), and were incubated with the corresponding horse radish peroxidase-labeled secondary antibody at the dilution of $1: 5000$ at $37^{\circ} \mathrm{C}$ for 1 hour. Western blots were visualized using 
Immobilon Western Chemiluminescent HRP Substrate (Merck Millipore, Burlington, MA, USA) followed by film exposure. $\beta$-actin was used as the internal reference. The gray level was analyzed, and the results were calculated as the gray level of the target protein divided by the gray level of the internal reference.

\section{Statistical analysis}

Statistical analysis was performed with Graphpad Prism software 7.00. Continuous data were calculated as mean \pm standard deviation (SD) and statistical significance was defined as $p<0.05$. Statistically significant differences among experimental groups were determined with student' t-test.

\section{Results}

\section{Combination of ASA ( $2 \mathrm{mmol} / \mathrm{L})$ and Gemcitabine (0.05 $\mathrm{mg} / \mathrm{L}$ ) Achieved More Inhibition in Cell Proliferation Compared with ASA or Gemcitabine Alone.}

As shown in Figure 1, the survival rate of SW1990 has more rapid reduction after treated with combination of ASA $(2 \mathrm{mmol} / \mathrm{L})$ and gemcitabine $(0.05 \mathrm{mg} / \mathrm{L})$ than with gemcitabine or ASA alone $(p<0.05)$. Similar results were observed with BxPC3 cells, showing the combination of ASA and gemcitabine inhibit achieved more inhibition in cell proliferation compared with ASA or gemcitabine alone. Collectively, the ASA regulates gemcitabine resistance in SW1990 and BxPC3 cells.

\section{Combination of ASA ( $2 \mathrm{mmol} / \mathrm{L})$ and Gemcitabine (0.05 $\mathrm{mg} / \mathrm{L}$ ) Inhibited Pancreatic Cell Migration.}

The wound healing assay was performed to validate the effect of ASA and gemcitabine on cell Migration. The migration effect was significantly reduced in cells treated with the combination of ASA and gemcitabine than with either ASA or gemcitabine alone $(p<0.05)$ (Figure 2), indicating the treatment with combination of ASA and gemcitabine depressed the mobility of SW1990 and BxPC3 pancreatic cancer cells.

\section{Effect of the Combination of ASA ( $2 \mathrm{mmol} / \mathrm{L})$ and Gemcitabine $(0.05 \mathrm{mg} / \mathrm{L})$ on Apoptosis of Pancreatic Cancer Cells.}

The proportion of apoptosis of the two pancreatic cancer cell lines SW1990 and BxPC3 was significantly higher when treated with the combination of ASA and gemcitabine than with ASA or gemcitabine alone $(p<0.05)$ (Fig. $3 A$ ). In control cells, the percentages of apoptosis were $4.13 \pm 0.88 \%$ and $4.79 \pm 0.65 \%$, 
respectively. In cells treated with ASA alone, these values were $7.84 \pm 2.35 \%$ and $6.12 \pm 0.23 \%$, respectively. In cells treated with gemcitabine alone, these values were $24.68 \pm 1.53 \%$ and $7.31 \pm 0.83 \%$, respectively. In cells treated with the combination of ASA and gemcitabine, these values were $32.13 \pm 1.95 \%$ and $11.32 \pm 0.98 \%$, respectively. Furthermore, the protein level of Bcl-2 was significantly more dramatically reduced when Bax was increased in those cells treated with the combination of ASA and gemcitabine than with either ASA or gemcitabine alone $(p<0.05)$ (Fig. 3B). Consistent with the aforementioned experimental results, the combination of ASA and gemcitabine contributes to apoptosis of pancreatic cancer cells more than ASA or gemcitabine alone. These evidences suggested that the ASA may promote antitumor effect of gemcitabine in SW1990 and BxPC3 cells.

\section{The Combination of ASA ( $2 \mathrm{mmol} / \mathrm{L})$ and Gemcitabine $(0.05 \mathrm{mg} / \mathrm{L})$ Increased Significantly the Expression of E- cadehrin and Decreased the Expression of Vimentin.}

Expression of EMT biomarkers in pancreatic cancer cells in vitro was significantly increased for Ecadehrin but decreased for Vimentin in the combination of ASA and gemcitabine group than in ASA or gemcitabine alone group $(p<0.05)$, indicating cell apoptosis and growth inhibition induced by the combination treatment may be closely related to EMT (Figure 4).

\section{The Combination of ASA ( $2 \mathrm{mmol} / \mathrm{L})$ and Gemcitabine $(0.05 \mathrm{mg} / \mathrm{L})$ Downregulated the PI3K/Akt/mTOR Signaling Pathway.}

Cells presented a significant decrease in the expression of p-PI3K and p-AKT after treated with combination of ASA ( $2 \mathrm{mmol} / \mathrm{L})$ and gemcitabine $(0.05 \mathrm{mg} / \mathrm{L})$ for $24 \mathrm{~h}$, compared with those observed in control and ASA or gemcitabine alone. Concurrently, the level of p-mTOR protein of the combination treatment group was depressed, measured through western blotting. These findings suggested that the downregulation of the PI3K/Akt/mTOR pathway may be partly involved in cell apoptosis and growth inhibition induced by the combination treatment (Figure 5).

\section{Discussion}

ASA, a widely used non-steroidal anti-inflammatory agent, has been reported to inhibit pancreatic cancer development by inhibition of mTOR signaling pathway, supporting the results we observed [27]. Regular use of ASA has been previously observed to be related to decreasing the risk of various types of cancer, such as Hepatocellular Carcinoma and breast cancer $[28,29]$. Recent research found that ASA inhibited GSK-3 $\beta$ activation on Capan-1 and PANC-1 when combining with gemcitabine [30]. Another recent study demonstrated that the combination of ASA and metformin significantly inhibited pancreatic cancer cell 
growth in vitro and in vivo by regulating the pro- and anti-apoptotic $\mathrm{Bcl}-2$ gene family members [31]. There are studies showed that low-dose ASA has a moderate chemopreventive effect on adenomas in the large bowel. Daily use of ASA is associated with a significant reduction in the incidence of colorectal adenomas in patients with previous colorectal cancer [32,33]. Recent research has also demonstrated that ASA can reduce the risk of cancer initiation and progression and can be used to suppress several tumor properties, including tumor cell migration [34,35]. However, no detailed study has been published, to the best of our knowledge, to assess the effect of ASA on gemcitabine monotherapy-related chemotherapy in pancreatic cancer cells. In our study, which concentration of ASA could be most suitable was a issue we discussed a lot. On the basis of the study of Shengping Lin, compared with untreated cells, PANC-1 cells treated with $2 \mathrm{mM}$ aspirin had a proliferation inhibition rate of about $40 \%$ at $24 \mathrm{~h}$ $(p<0.05)$ [36]. We chose the $2 \mathrm{mmol} / \mathrm{L}$ concentration of ASA as a new attempt. However, compared with some reports, $2 \mathrm{mmol} / \mathrm{L}$ concentration seems to be a bit higher. We speculated it might be that the concentration of aspirin in pancreatic cancer is inconclusive and the concentration of aspirin in other tumors may be different from that in pancreatic cancer. What's more, overexpressed extracellular matrix (ECM) in pancreatic cancer limits drug penetration into the tumor and is associated with concentration of ASA in pancreatic cancer microenvironment. However, researchers have been looking for different ways. It's reported collagenase nanoparticles could enhance the penetration of drugs into pancreatic cancer. Research declared that a pretreatment based on a proteolytic-enzyme nanoparticle system disassembles the dense pancreatic cancer collagen stroma and increases drug penetration into the pancreatic cancer [37]. Moreover, to address the problem of drug penetration, some researchers demonstrated a dendrimer-camptothecin (CPT) conjugate that actively penetrates deep into pancreatic cancer through $\mathrm{Y}^{-}$ glutamyl transpeptidase (GGT)-triggered cell endocytosis and transcytosis. This dendrimer-drug conjugate even exhibited high antitumor activity in multiple mice tumor models compared to the standard first-line chemotherapeutic drug (gemcitabine) for advanced pancreatic cancer [38]. To sum up, increase the concentration of drugs in pancreatic cancer microenvironment requires the progress of materials science, biology, pharmacology and other fields.

The role of ASA therapy in the tumorigenesis and development of pancreatic cancer and the underlying mechanism remain unclear. The goal of this study was to validate whether ASA could help to inhibit gemcitabine resistance so that more patients are able to respond to gemcitabine. In addition, we looked for the possible molecules involved in gemcitabine resistance.

Previous research has addressed the role of EMT in tumor cell drug resistance [39]. Therefore, the mechanisms of EMT in pancreatic cancer needs research for the development of new therapeutic strategies [40]. Our study showed that ASA could increase the expression level of E-cadherin and decrease in the expression level of Vimentin in SW1990 and BxPC3, indicating ASA could inhibit gemcitabine resistance of pancreatic cancer cells through transformed EMT.

The PI3K/Akt family is among the most frequently mutated pathways in human cancer. Aberrant activation of the PI3K/Akt signaling pathway is thought to be associated with development of chemotherapeutic resistance. Multiple genetic events have been described that lead to activation of the 
$\mathrm{PI3K} / A k t / m T O R$ pathway in cancer [41]. Akt/mTOR pathways inhibitor like rapamycin and LY294002 could inhibit the EMT progression in pancreatic cancer. Study shows that ROS is concidered to regulate EMT [42]. ROS balance cellular integrity and cell death while several anticancer drugs have been shown to induce high levels of ROS and decrease of AKT/mTOR signaling pathway[43-45]. Our study showed that ASA could inhibit gemcitabine resistance of pancreatic cancer cells through downregulated $\mathrm{PI} 3 \mathrm{~K} / \mathrm{Akt} / \mathrm{mTOR}$ (e.g. p-PI3K, p-AKT and p-mTOR), which are consistent with previous reports demonstrating that gemcitabine inhibited pancreatic cancer growth and metastasis and that gemcitabine inhibited EMT with the involvement of PI3K/Akt/mTOR pathway in pancreatic cancer cells [46]. We also have found that the combination of ASA and gemcitabine exerts a remarkable inducing effect on cell proliferation and markedly promotes apoptosis in both SW1990 and BXPC3 cell lines with the involvement of PI3K/Akt/mTOR signaling pathway. Between the 2 cell lines used in our study, the difference in response rates deserves discussion. Throughout a large number of literature basis, the difference in response rates between the BxPC3 and SW1990 cell line is existent. Some researchers pointed out when treated with gemcitabine, SW1990 cell line's IC50 is $6.266 \mathrm{mmol} / \mathrm{L}$ while BXPC3 cell line's is $4.01 \mathrm{mmol} / \mathrm{L}$ [47]. What's clear is that, when treated with gemcitabine, the BxPC3 cell line may has stronger drug resistance in some malignant biological behaviors.

What we found is that low-dose ASA combined with gemcitabine significantly reduced cell proliferation, inhibited cell migration, increased the apoptosis, increased expression of E-cadherin and suppressed that of Vimentin, when compared with gemcitabine alone. When ASA plus gemcitabine therapy compared with ASA or gemcitabine alone, the result showed significant downregulation of the PI3K/AKT/mTOR pathway on SW1990 and BXPC3 cells, as indicated by effectively reducing the phosphorylation of PI3K, Akt and mTOR. These data suggested that Synergistic effect of ASA plus gemcitabine therapy in pancreatic cancer may occured by reversing EMT via downregulation of the PI3K/AKT/mTOR signaling pathway. These results are similar to those seen in a previous study, in which ASA was found to inhibit the EMT and migration of oncogenic K-ras-expressing non-small cell lung carcinoma cells by downregulating the E-cadherin repressor Slug [48].

In summary, our results demonstrated that ASA could help to inhibit gemcitabine resistance, which induced more inhibition in cell proliferation, reduction in migration and increase in apoptosis. Our work also revealed that ASA could regulate gemcitabine resistance by inhibiting the PI3K/Akt/mTOR signaling pathway to reverse EMT in pancreatic cancer cells, where we provided new insight into. The results provide a promising new therapeutic option for pancreatic carcinoma patients.

\section{Declarations}

\section{Ethics approval and consent to participate}

Not applicable. 


\section{Consent for publication}

All authors have read and approved the final manuscript.

\section{Availability of data and materials}

The datasets used and/or analyzed during the current study are available from the corresponding author on reasonable request.

\section{Competing interests}

All authors declare that they have no competing interests.

\section{Funding}

This work was supported by grants from Project of Changzhou Science and technology Bureau (ZD201711), CSCO-Henrui Cancer Research Fundation (Y-HR2017-014).

\section{Authors' contributions}

Hanyu Zhou performed experiments. ZhiYing Yan, Feifei Wei and Xiao Yun analyzed the data. MuHan Liu wrote the manuscript. Yi Liu and Xianming Zhang analyzed the data of cell apoptosis. Kequn Xu and Yingwei Zhu edited the manuscript. All authors have read and approved the final manuscript.

\section{Acknowledgements}

The authors acknowledge that Can Hou made suggestions for revision.

\section{References}

1. R. L. Siegel, K. D. MillerA. Jemal. Cancer statistics, 2020. CA Cancer J Clin. 2020;70(1):7-30. doi: $10.3322 /$ caac. 21590

2. D. A. TuvesonJ. P. Neoptolemos. Understanding metastasis in pancreatic cancer: a call for new clinical approaches. Cell. 2012;148(1-2):21-3. doi: 10.1016/j.cell.2011.12.021

3. Z. Wang, Y. Li, A. Ahmad, S. Banerjee, A. S. Azmi, D. Kong, et al. Pancreatic cancer: understanding and overcoming chemoresistance. Nat Rev Gastroenterol Hepatol. 2011;8(1):27-33. doi:

10.1038/nrgastro.2010.188 
4. M. Tang, D. Svirskis, E. Leung, M. Kanamala, H. WangZ. Wu. Can intracellular drug delivery using hyaluronic acid functionalised $\mathrm{pH}$-sensitive liposomes overcome gemcitabine resistance in pancreatic cancer? J Control Release. 2019;305(89-100. doi: 10.1016/j.jconrel.2019.05.018

5. C. J. Halbrook, C. Pontious, I. Kovalenko, L. Lapienyte, S. Dreyer, H. J. Lee, et al. MacrophageReleased Pyrimidines Inhibit Gemcitabine Therapy in Pancreatic Cancer. Cell Metab. 2019;29(6):1390-1399 e6. doi: 10.1016/j.cmet.2019.02.001

6. K. M. Mann, H. Ying, J. Juan, N. A. JenkinsN. G. Copeland. KRAS-related proteins in pancreatic cancer. Pharmacol Ther. 2016;168(29-42. doi: 10.1016/j.pharmthera.2016.09.003

7. M. Song, A. M. Bode, Z. DongM. H. Lee. AKT as a Therapeutic Target for Cancer. Cancer Res. 2019;79(6):1019-1031. doi: 10.1158/0008-5472.CAN-18-2738

8. R. J. ShawL. C. Cantley. Ras, $\mathrm{PI}(3) \mathrm{K}$ and mTOR signalling controls tumour cell growth. Nature. 2006;441(7092):424-30. doi: 10.1038/nature04869

9. Y. Zhang, P. Kwok-Shing Ng, M. Kucherlapati, F. Chen, Y. Liu, Y. H. Tsang, et al. A Pan-Cancer Proteogenomic Atlas of PI3K/AKT/mTOR Pathway Alterations. Cancer Cell. 2017;31(6):820-832 e3. doi: 10.1016/j.ccell.2017.04.013

10. M. Feng, G. Xiong, Z. Cao, G. Yang, S. Zheng, J. Qiu, et al. LAT2 regulates glutamine-dependent mTOR activation to promote glycolysis and chemoresistance in pancreatic cancer. J Exp Clin Cancer Res. 2018;37(1):274. doi: 10.1186/s13046-018-0947-4

11. P. Zhou, B. Li, F. Liu, M. Zhang, Q. Wang, Y. Liu, et al. The epithelial to mesenchymal transition (EMT) and cancer stem cells: implication for treatment resistance in pancreatic cancer. Mol Cancer. 2017;16(1):52. doi: 10.1186/s12943-017-0624-9

12. R. Marais, W. Sellers, D. LivingstonE. Mihich. Twenty-fourth annual Pezcoller symposium: Molecular basis for resistance to targeted agents. Cancer Res. 2013;73(3):1046-9. doi: 10.1158/00085472.CAN-12-3236

13. B. De Craene, B. Gilbert, C. Stove, E. Bruyneel, F. van RoyG. Berx. The transcription factor snail induces tumor cell invasion through modulation of the epithelial cell differentiation program. Cancer Res. 2005;65(14):6237-44. doi: 10.1158/0008-5472.CAN-04-3545

14. S. Lamouille, J. XuR. Derynck. Molecular mechanisms of epithelial-mesenchymal transition. Nat Rev Mol Cell Biol. 2014;15(3):178-96. doi: 10.1038/nrm3758

15. S. Y. Chaw, A. Abdul Majeed, A. J. Dalley, A. Chan, S. SteinC. S. Farah. Epithelial to mesenchymal transition (EMT) biomarkers--E-cadherin, beta-catenin, APC and Vimentin-in oral squamous cell carcinogenesis and transformation. Oral Oncol. 2012;48(10):997-1006. doi: 10.1016/j.oraloncology.2012.05.011

16. T. Luo, L. Wang, P. Wu, W. Gong, W. Chen, H. Zhao, et al. Downregulated vimentin and upregulated Ecadherin in T1 stage non-small-cell lung cancer: does it suggest a mesenchymal-epithelial transition? Neoplasma. 2017;64(5):693-699. doi: 10.4149/neo_2017_506

17. W. Wang, L. Wang, A. Mizokami, J. Shi, C. Zou, J. Dai, et al. Down-regulation of E-cadherin enhances prostate cancer chemoresistance via Notch signaling. Chin J Cancer. 2017;36(1):35. doi: 
10.1186/s40880-017-0203-x

18. P. Mallini, T. Lennard, J. KirbyA. Meeson. Epithelial-to-mesenchymal transition: what is the impact on breast cancer stem cells and drug resistance. Cancer Treat Rev. 2014;40(3):341-8. doi: 10.1016/j.ctrv.2013.09.008

19. S. Zelenay, A. G. van der Veen, J. P. Bottcher, K. J. Snelgrove, N. Rogers, S. E. Acton, et al. Cyclooxygenase-Dependent Tumor Growth through Evasion of Immunity. Cell. 2015;162(6):1257-70. doi: 10.1016/j.cell.2015.08.015

20. Y. Ergun, N. Y. Ozdemir, E. K. Guner, E. Esin, M. A. Sendur, E. B. Koksoy, et al. Comparison of Gemcitabine monotherapy with Gemcitabine and Cisplatin combination in metastatic pancreatic cancer: a retrospective analysis. J BUON. 2018;23(7):116-121. doi:

21. W. Li, Y. Zhu, K. Zhang, X. Yu, H. Lin, W. Wu, et al. PROM2 promotes gemcitabine chemoresistance via activating the Akt signaling pathway in pancreatic cancer. Exp Mol Med. 2020;52(3):409-422. doi: 10.1038/s12276-020-0390-4

22. R. Wei, N. E. C. Penso, R. M. Hackman, Y. WangG. G. Mackenzie. Epigallocatechin-3-Gallate (EGCG) Suppresses Pancreatic Cancer Cell Growth, Invasion, and Migration partly through the Inhibition of Akt Pathway and Epithelial-Mesenchymal Transition: Enhanced Efficacy when Combined with Gemcitabine. Nutrients. 2019;11(8). doi: 10.3390/nu11081856

23. T. Namba, R. Kodama, S. Moritomo, T. HoshinoT. Mizushima. Zidovudine, an anti-viral drug, resensitizes gemcitabine-resistant pancreatic cancer cells to gemcitabine by inhibition of the AktGSK3beta-Snail pathway. Cell Death Dis. 2015;6(e1795. doi: 10.1038/cddis.2015.172

24. C. M. Ulrich, J. BiglerJ. D. Potter. Non-steroidal anti-inflammatory drugs for cancer prevention: promise, perils and pharmacogenetics. Nat Rev Cancer. 2006;6(2):130-40. doi: 10.1038/nrc1801

25. P. M. Rothwell, F. G. Fowkes, J. F. Belch, H. Ogawa, C. P. WarlowT. W. Meade. Effect of daily aspirin on long-term risk of death due to cancer: analysis of individual patient data from randomised trials. Lancet. 2011;377(9759):31-41. doi: 10.1016/S0140-6736(10)62110-1

26. X. L. Tan, K. M. Reid Lombardo, W. R. Bamlet, A. L. Oberg, D. P. Robinson, K. E. Anderson, et al. Aspirin, nonsteroidal anti-inflammatory drugs, acetaminophen, and pancreatic cancer risk: a clinic-based case-control study. Cancer Prev Res (Phila). 2011;4(11):1835-41. doi: 10.1158/1940-6207.CAPR-110146

27. W. Yue, C. S. Yang, R. S. DiPaolaX. L. Tan. Repurposing of metformin and aspirin by targeting AMPKmTOR and inflammation for pancreatic cancer prevention and treatment. Cancer Prev Res (Phila). 2014;7(4):388-97. doi: 10.1158/1940-6207.CAPR-13-0337

28. T. G. Simon, Y. Ma, J. F. Ludvigsson, D. Q. Chong, E. L. Giovannucci, C. S. Fuchs, et al. Association Between Aspirin Use and Risk of Hepatocellular Carcinoma. JAMA Oncol. 2018;4(12):1683-1690. doi: 10.1001/jamaoncol.2018.4154

29. R. D. Kehm, J. L. Hopper, E. M. John, K. A. Phillips, R. J. Maclnnis, G. S. Dite, et al. Regular use of aspirin and other non-steroidal anti-inflammatory drugs and breast cancer risk for women at familial or genetic risk: a cohort study. Breast Cancer Res. 2019;21(1):52. doi: 10.1186/s13058-019-1135-y 
30. Y. Q. Ou, W. Zhu, Y. Li, P. X. Qiu, Y. J. Huang, J. Xie, et al. Aspirin inhibits proliferation of gemcitabineresistant human pancreatic cancer cells and augments gemcitabine-induced cytotoxicity. Acta Pharmacol Sin. 2010;31(1):73-80. doi: 10.1038/aps.2009.172

31. W. Yue, X. Zheng, Y. Lin, C. S. Yang, Q. Xu, D. Carpizo, et al. Metformin combined with aspirin significantly inhibit pancreatic cancer cell growth in vitro and in vivo by suppressing anti-apoptotic proteins Mcl-1 and Bcl-2. Oncotarget. 2015;6(25):21208-24. doi: 10.18632/oncotarget.4126

32. J. A. Baron, B. F. Cole, R. S. Sandler, R. W. Haile, D. Ahnen, R. Bresalier, et al. A randomized trial of aspirin to prevent colorectal adenomas. N Engl J Med. 2003;348(10):891-9. doi: 10.1056/NEJMoa021735

33. R. S. Sandler, S. Halabi, J. A. Baron, S. Budinger, E. Paskett, R. Keresztes, et al. A randomized trial of aspirin to prevent colorectal adenomas in patients with previous colorectal cancer. $\mathrm{N}$ Engl $\mathrm{J}$ Med. 2003;348(10):883-90. doi: 10.1056/NEJMoa021633

34. Y. Cao, R. Nishihara, K. Wu, M. Wang, S. Ogino, W. C. Willett, et al. Population-wide Impact of Longterm Use of Aspirin and the Risk for Cancer. JAMA Oncol. 2016;2(6):762-9. doi:

10.1001/jamaoncol.2015.6396

35. D. M. Fraser, F. M. Sullivan, A. M. ThompsonC. McCowan. Aspirin use and survival after the diagnosis of breast cancer: a population-based cohort study. Br J Cancer. 2014;111(3):623-7. doi: 10.1038/bjc.2014.264

36. S. Lin, Y. PanC. Xu. Effects of aspirin on pancreatic cancer cells PANC-1 and its potential molecular mechanism. J BUON. 2020;25(5):2449-2455. doi:

37. A. Zinger, L. Koren, O. Adir, M. Poley, M. Alyan, Z. Yaari, et al. Collagenase Nanoparticles Enhance the Penetration of Drugs into Pancreatic Tumors. ACS Nano. 2019;13(10):11008-11021. doi: $10.1021 /$ acsnano.9b02395

38. G. Wang, Z. Zhou, Z. Zhao, Q. Li, Y. Wu, S. Yan, et al. Enzyme-Triggered Transcytosis of DendrimerDrug Conjugate for Deep Penetration into Pancreatic Tumors. ACS Nano. 2020;14(4):4890-4904. doi: 10.1021/acsnano.0c00974

39. F. Zhu, J. Kai, L. Chen, M. Wu, J. Dong, Q. Wang, et al. Akt Inhibitor Perifosine Prevents Epileptogenesis in a Rat Model of Temporal Lobe Epilepsy. Neurosci Bull. 2018;34(2):283-290. doi: 10.1007/s12264-017-0165-7

40. T. Arumugam, V. Ramachandran, K. F. Fournier, H. Wang, L. Marquis, J. L. Abbruzzese, et al. Epithelial to mesenchymal transition contributes to drug resistance in pancreatic cancer. Cancer Res. 2009;69(14):5820-8. doi: 10.1158/0008-5472.CAN-08-2819

41. L. M. Thorpe, H. YuzugulluJ. J. Zhao. PI3K in cancer: divergent roles of isoforms, modes of activation and therapeutic targeting. Nat Rev Cancer. 2015;15(1):7-24. doi: 10.1038/nrc3860

42. E. L. Samuel, D. C. Marcano, V. Berka, B. R. Bitner, G. Wu, A. Potter, et al. Highly efficient conversion of superoxide to oxygen using hydrophilic carbon clusters. Proc Natl Acad Sci U S A. 2015;112(8):23438. doi: $10.1073 /$ pnas. 1417047112 
43. D. Trachootham, W. Lu, M. A. Ogasawara, R. D. NilsaP. Huang. Redox regulation of cell survival. Antioxid Redox Signal. 2008;10(8):1343-74. doi: 10.1089/ars.2007.1957

44. K. He, L. Wu, Q. Ding, F. Haider, H. Yu, H. Wang, et al. Apatinib Promotes Apoptosis of Pancreatic Cancer Cells through Downregulation of Hypoxia-Inducible Factor-1alpha and Increased Levels of Reactive Oxygen Species. Oxid Med Cell Longev. 2019;2019(5152072. doi: 10.1155/2019/5152072

45. P. Zou, J. Zhang, Y. Xia, K. Kanchana, G. Guo, W. Chen, et al. ROS generation mediates the anti-cancer effects of WZ35 via activating JNK and ER stress apoptotic pathways in gastric cancer. Oncotarget. 2015;6(8):5860-76. doi: 10.18632/oncotarget.3333

46. F. Wang, Q. Wang, Z. W. Zhou, S. N. Yu, S. T. Pan, Z. X. He, et al. Plumbagin induces cell cycle arrest and autophagy and suppresses epithelial to mesenchymal transition involving PI3K/Akt/mTORmediated pathway in human pancreatic cancer cells. Drug Des Devel Ther. 2015;9(537-60. doi: 10.2147/DDDT.S73689

47. W. J. GuH. L. Liu. Induction of pancreatic cancer cell apoptosis, invasion, migration, and enhancement of chemotherapy sensitivity of gemcitabine, 5-FU, and oxaliplatin by hnRNP A2/B1 siRNA. Anticancer Drugs. 2013;24(6):566-76. doi: 10.1097/CAD.0b013e3283608bc5

48. D. A. Drew, Y. CaoA. T. Chan. Aspirin and colorectal cancer: the promise of precision chemoprevention. Nat Rev Cancer. 2016;16(3):173-86. doi: 10.1038/nrc.2016.4

\section{Figures}

SW1990

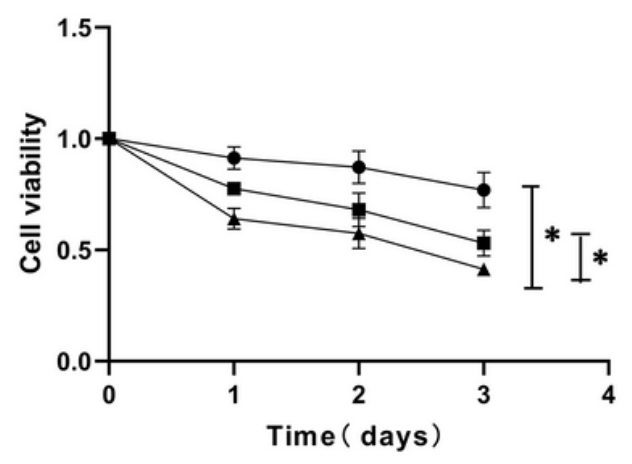

BxPC3

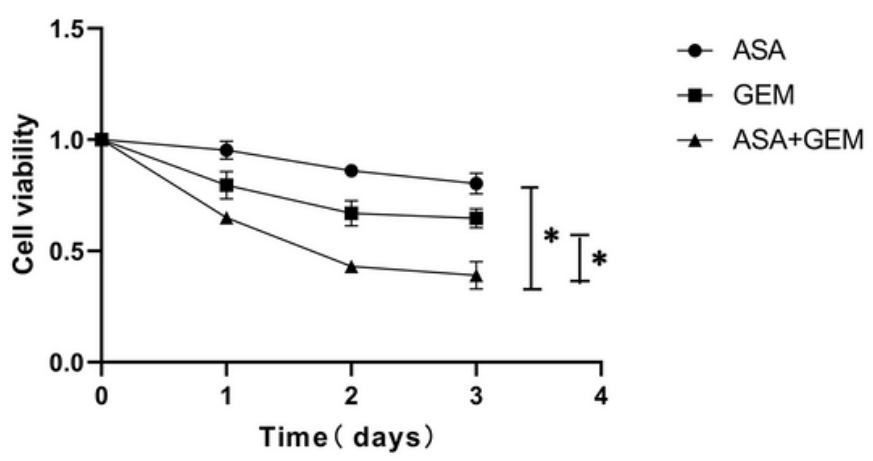

Figure 1

Combination of ASA ( $2 \mathrm{mmol} / \mathrm{L})$ and gemcitabine $(0.05 \mathrm{mg} / \mathrm{L})$ achieved more inhibition in cell proliferation compared with ASA or gemcitabine alone. The SW1990 and BXPC3 cells were treated with ASA ( $2 \mathrm{mmol} / \mathrm{L})$ and/or gemcitabine $(0.05 \mathrm{mg} / \mathrm{L})$ for different time intervals. The inhibitory activity was expressed as an inhibition rate. All experiments were performed in triplicate. The values are expressed as mean \pm SD of three replicates, ${ }^{\star} \mathrm{P}<0.05$. 
SW1990
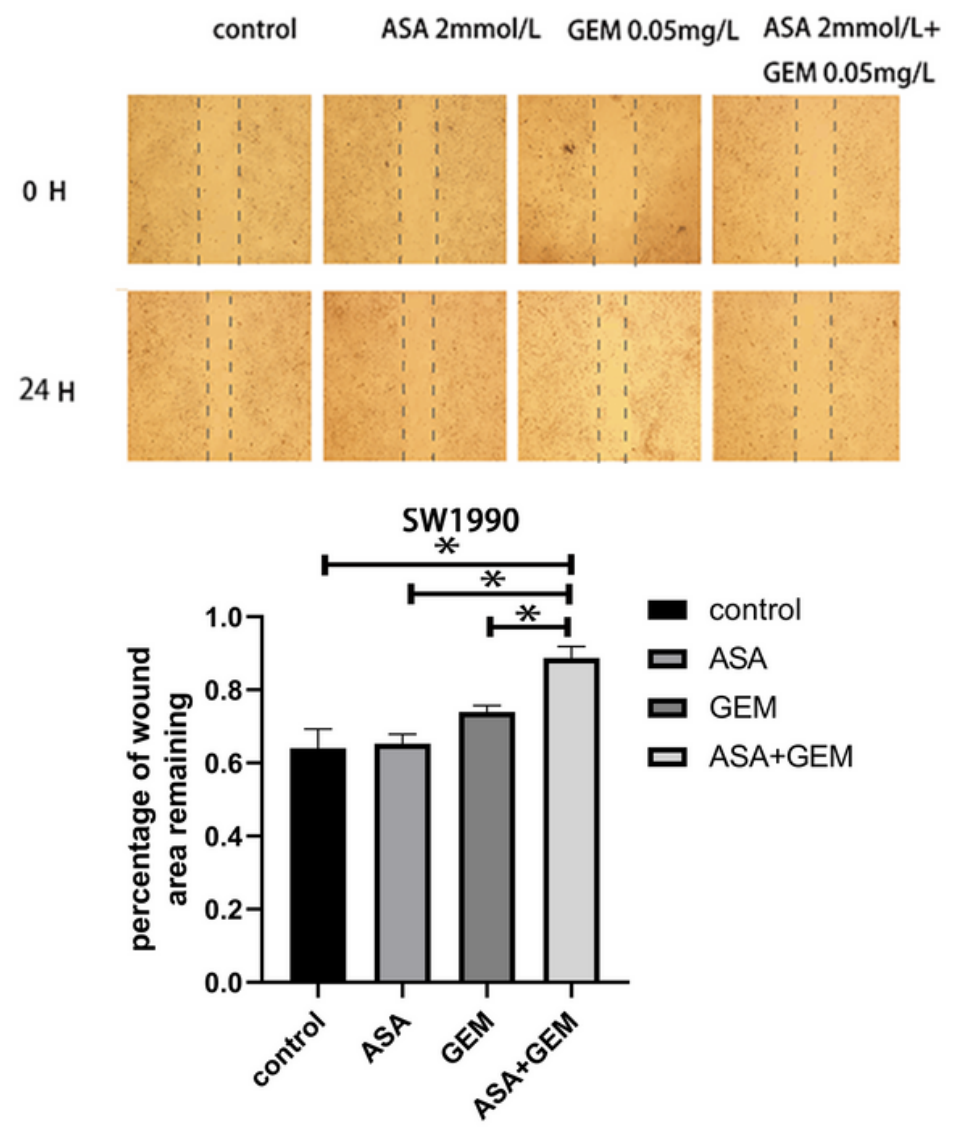

$\mathrm{BxPC3}$

control ASA $2 \mathrm{mmol} / \mathrm{L} \quad$ GEM $0.05 \mathrm{mg} / \mathrm{L} \quad$ ASA $2 \mathrm{mmol} / \mathrm{L}+$ GEM $0.05 \mathrm{mg} / \mathrm{L}$

$\mathrm{OH}$

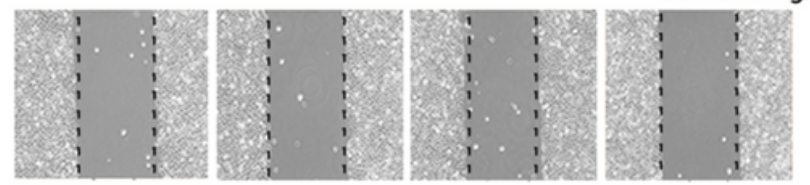

$24 \mathrm{H}$
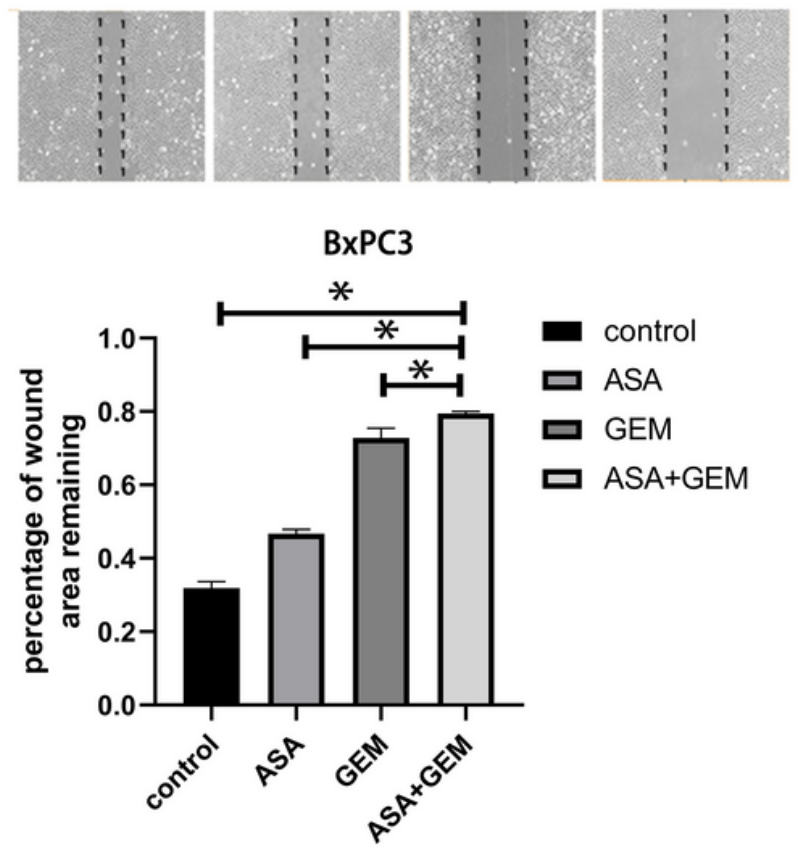

Figure 2

Combination of ASA $(2 \mathrm{mmol} / \mathrm{L})$ and gemcitabine $(0.05 \mathrm{mg} / \mathrm{L})$ inhibited pancreatic cell migration. The movement ability of SW1990 and BXPC3 cells after treated with ASA and/or gemcitabine for $24 \mathrm{~h}$ was detected using wound healing assays. We used electronic microscope (X400) to observe. The width(W) of the scratch measured; the percentage of wound area remaining was calculated as W24h/W0h $\times 100 \%$. All experiments were performed in triplicate. The results represent mean \pm standard deviation, ${ }^{\star} \mathrm{P}<0.05$. 
A

\section{Control}

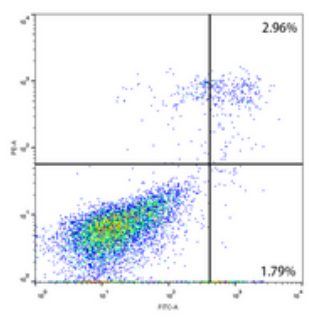

Control

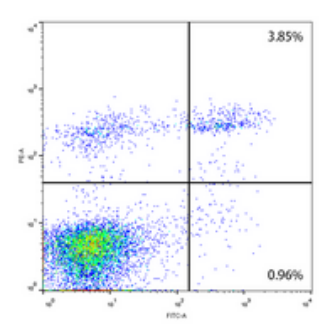

\section{SW1990}

ASA $2 \mathrm{mmol} / \mathrm{L}$
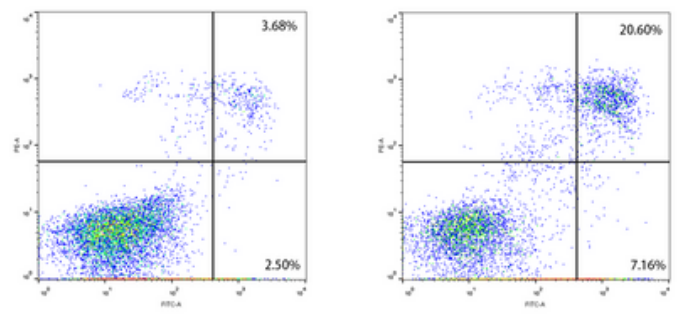

BxPC3

GEM $0.05 \mathrm{mg} / \mathrm{L}$

ASA $2 \mathrm{mmol} / \mathrm{L}+$ GEM $0.05 \mathrm{mg} / \mathrm{L}$
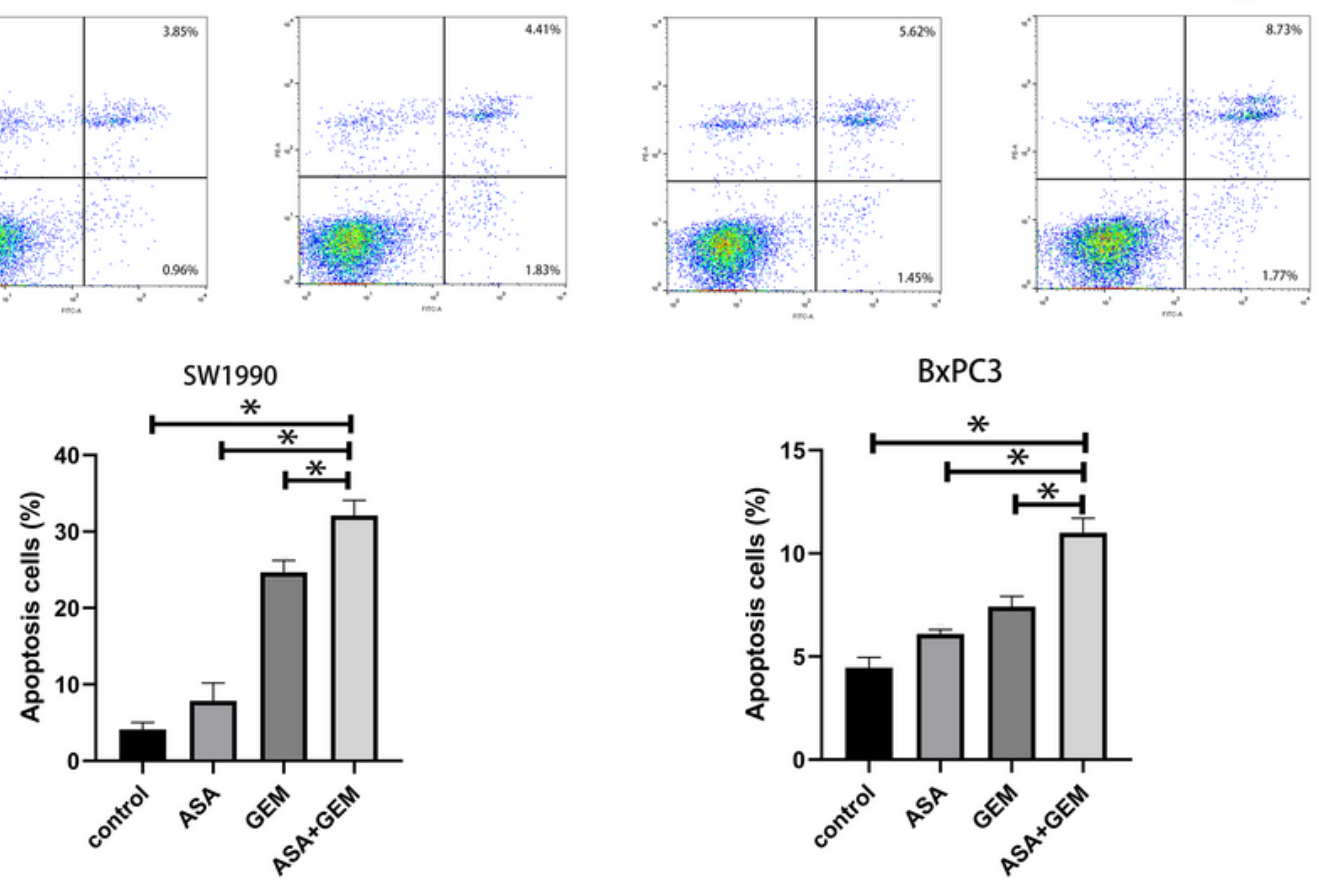

B
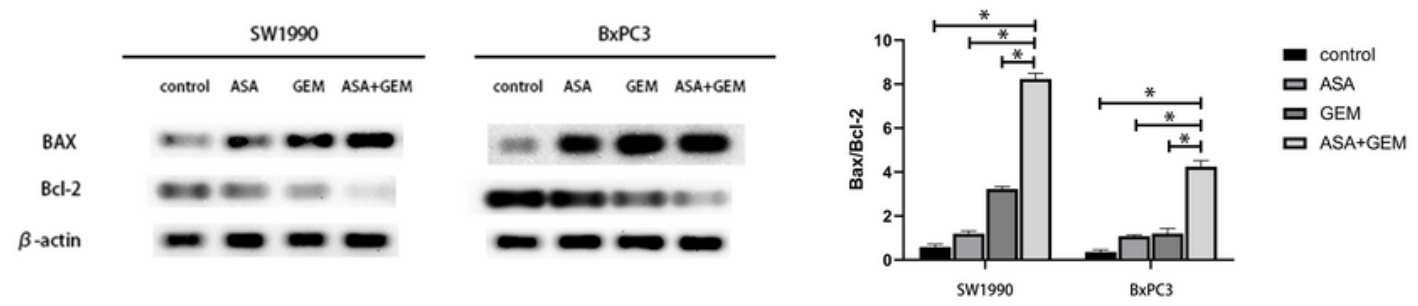

Figure 3

Effect of the combination of ASA ( $2 \mathrm{mmol} / \mathrm{L})$ and gemcitabine $(0.05 \mathrm{mg} / \mathrm{L})$ on apoptosis of pancreatic cancer cells. ASA plus gemcitabine increased pancreatic cancer cell apoptosis. Pancreatic cancer cell lines were treated with gemcitabine and/or ASA for 48 hours. (A) Annexin V-FITC/PI staining assay was used to detect cell apoptosis. The quantitative analysis of the apoptotic cell numbers is shown.

(B)Western blot assay was used to evaluate the expression of apoptosis-related proteins including Bcl-2 
and Bax. The data shown represent the mean \pm SD from 3 independent experiments with similar results. The bars represent mean \pm standard deviation, ${ }^{*} \mathrm{P}<0.05$.

control ASA GEM ASA+GEM

\section{E-cadherin}

Vimentin

$$
\beta \text {-actin }
$$

SW1990

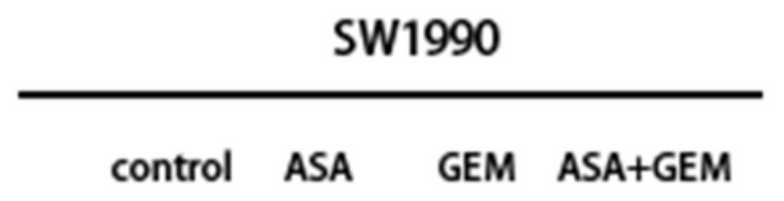

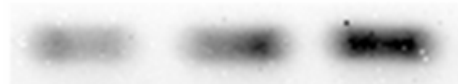

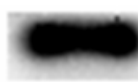

10

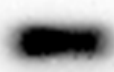

\section{(1)}

\section{BxPC3}
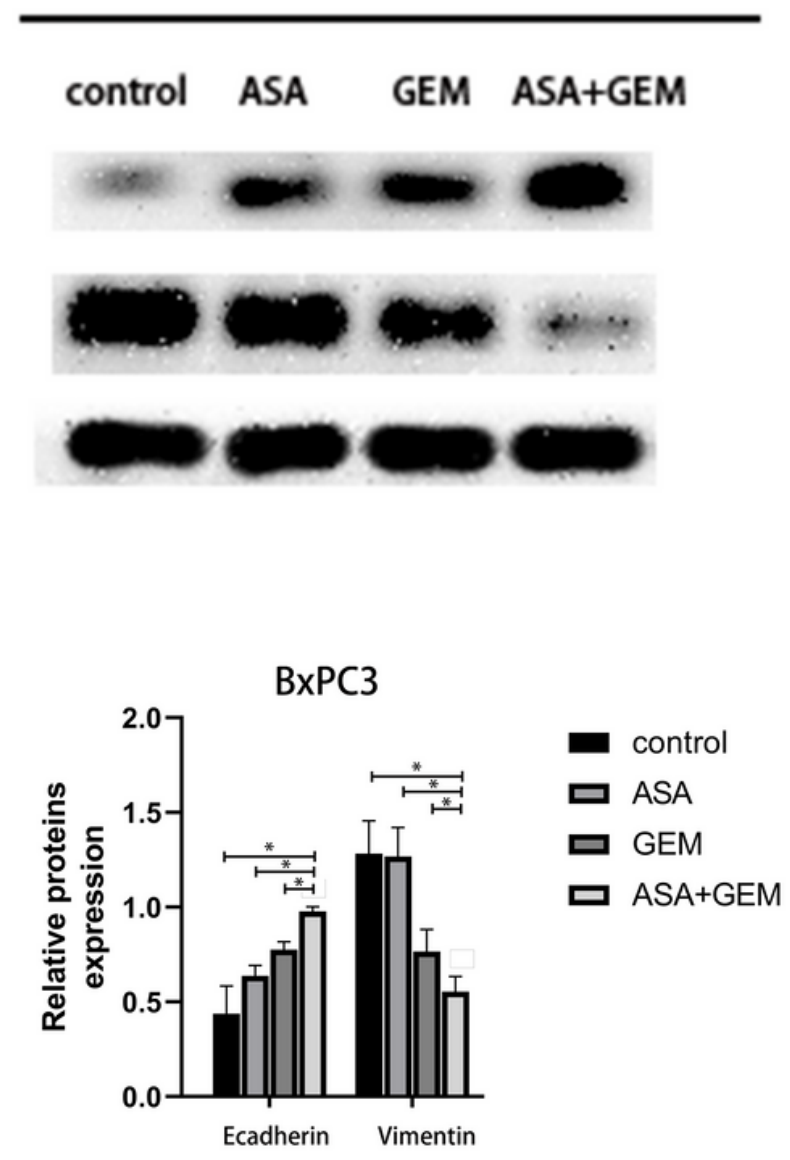

Figure 4

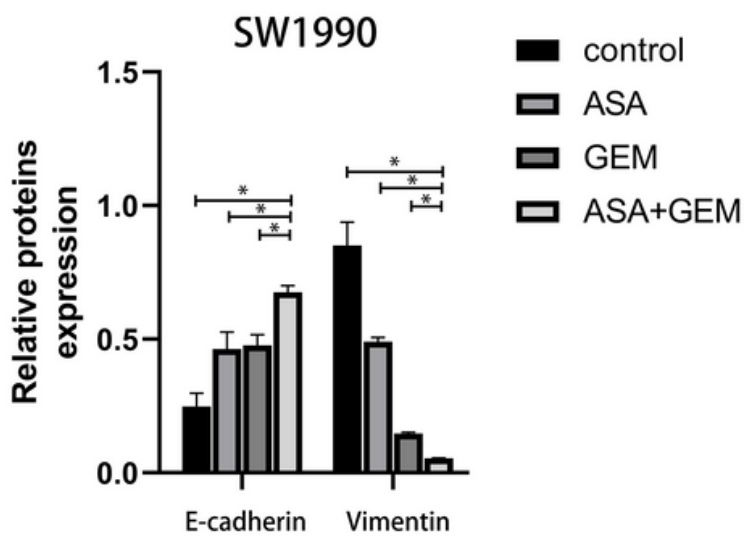

The combination of ASA $(2 \mathrm{mmol} / \mathrm{L})$ and gemcitabine $(0.05 \mathrm{mg} / \mathrm{L})$ increased significantly the expression of E-cadehrin and decreased the expression of Vimentin. SW1990 and BxPC3 cells were treated with ASA and/or gemcitabine for $24 \mathrm{~h}$ and the levels of EMT-related proteins(E-cadherin, Vimentin) were determined through western blotting while $\beta$-actin was included as a loading control. All experiments were performed in triplicate, ${ }^{*} \mathrm{P}<0.05$. 


\section{SW1990}

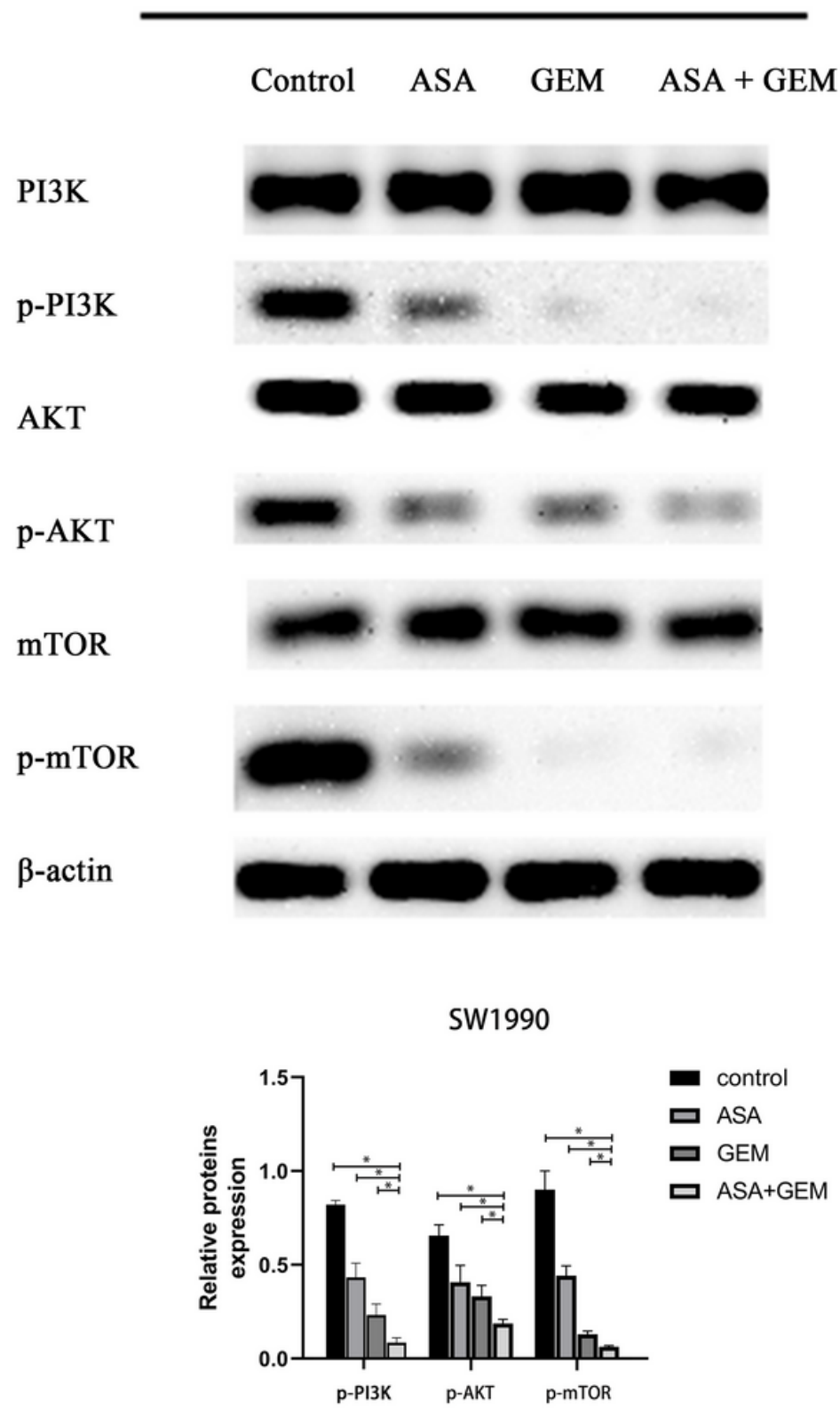

\section{$\mathrm{BxPC} 3$}

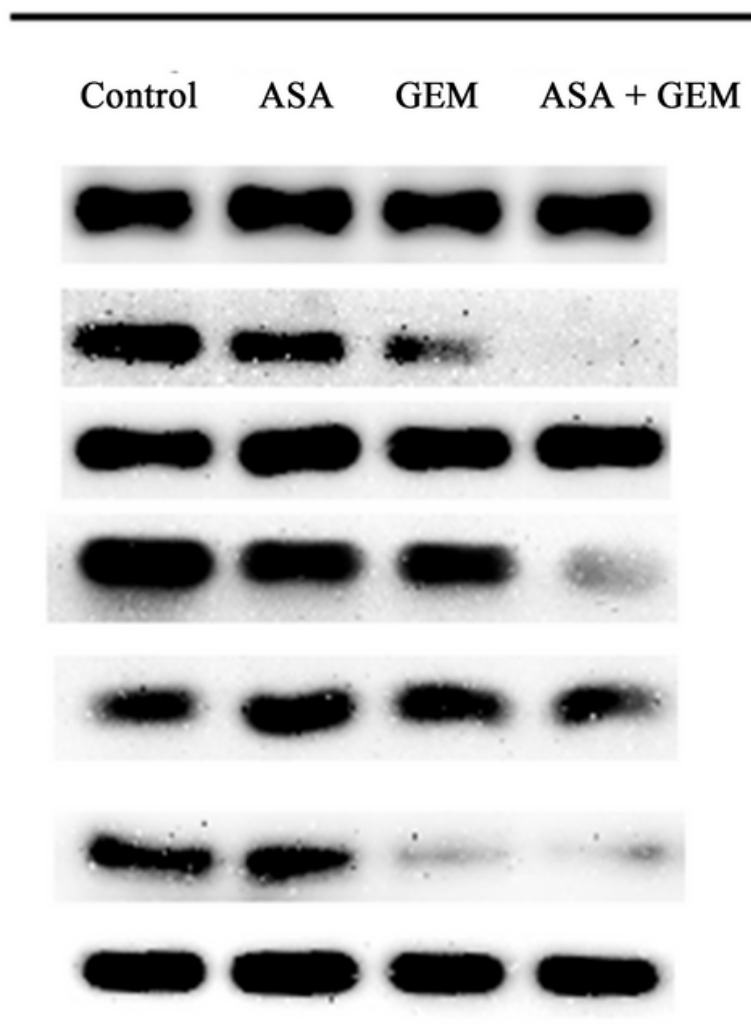

BxPC3

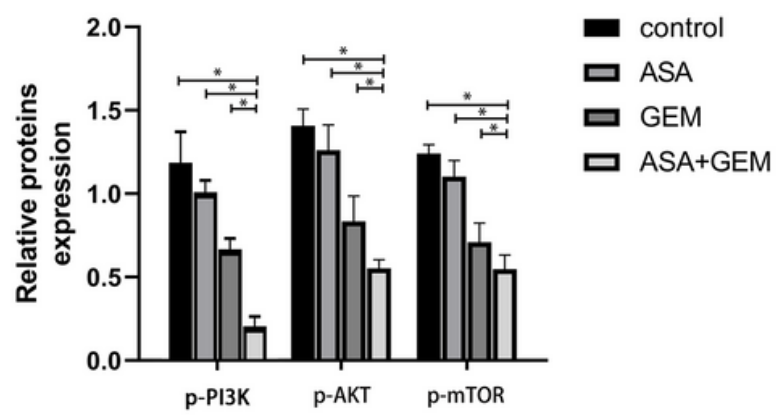

\section{Figure 5}

The combination of ASA ( $2 \mathrm{mmol} / \mathrm{L})$ and gemcitabine $(0.05 \mathrm{mg} / \mathrm{L})$ downregulated the PI3K/Akt/mTOR signaling pathway. SW1990 and BxPC3 cells were treated with ASA and/or gemcitabine for $24 \mathrm{~h}$ and the levels of PI3K, AKT, mTOR, phosphorylated PI3K, phosphorylated AKT and phosphorylated mTOR were determined through western blotting while $\beta$-actin was included as a loading control. All experiments were performed in triplicate, ${ }^{*} \mathrm{P}<0.05$. 